\title{
Lifestyle including dietary habits and changes in coronary artery calcium score: a retrospective cohort study
}

\author{
EunSun Cheong, Jong-Young Lee, Sung Ho Lee, Jin-Ho Kang, Bum-Soo Kim, Byung Jin Kim and Ki-Chul Sung*
}

\begin{abstract}
Background: General diet and lifestyle are considered to have an effect on levels of atherosclerosis but previous studies have shown inconsistent results. The aim of this study was to investigate whether macronutrient intake, physical activity and depressive symptoms are associated with progression of preclinical atherosclerosis in healthy Korean adults.

Methods: A total of 2623 individuals from Kangbuk Samsung Hospital Health Screening Center in South Korea were enrolled between 2010 and 2012 and had follow-up at 2013. Every participant received a non-enhanced coronary computed tomography (CT) and completed questionnaires for food intake frequency, depression and physical activity levels. The study population was divided into two groups according to CAC progression, namely CAC group (CAC score $>0$ ) or non-CAC group (CAC score $\leq 0$ ), and were compared according to macronutrient intake, degree of depressive symptoms and physical activity.
\end{abstract}

Results: A total of 2175 subjects were eligible for the analysis and among them, 592 subjects had progression of CAC. Total energy, carbohydrate and fat intake showed significant differences between the two groups ( $p$-values of $0.01,0.021$ and 0.016 respectively). However, levels of protein intake did not vary for the two groups $(p=0.286)$. Depressive symptoms and extent of physical activity were similar between the two groups. Multivariate analysis was conducted with adjustment for possible confounding factors. The hazard ratios for CAC progression were not different according to macronutrient intake, degree of depressive symptoms and physical activity.

Conclusion: In this large relatively healthy population based observational study, CAC progression showed no significant association with total energy intake, proportion of macronutrient intake, depressive symptom and physical activity.

Keywords: Dietary habits, Lifestyle, Coronary artery calcium score, Macronutrient, Physical activity, Depression

\section{Background}

Coronary artery calcification (CAC) is regarded as important pathognomonic sign of coronary atherosclerosis [1]. The presence of subintimal coronary calcification results from a healing process after rupture of unstable lesions from atheroma $[2,3]$ and measurement of CAC by multidetector computed tomography (MDCT) is widely used as a non-invasive tool

\footnotetext{
* Correspondence: kcmd.sung@samsung.com

Division of Cardiology, Department of Medicine, Kangbuk Samsung Hospital, Sungkyunkwan University, School of Medicine, \#108, Pyung Dong, Jongro-Ku, Seoul 110-746, Republic of Korea
}

for assessing atherosclerotic plaque burden in the coronary arteries.

Although age, sex, hypertension, dyslipidemia, diabetes, glucose intolerance, smoking, obesity are all known risk factors for developing $\mathrm{CAC}$, they are inconsistent predictors [4-7]. This suggests that other unrecognized risk factors may be present for developing CAC. It is plausible that dietary factors increase the sk of CAC and previous findings have shown that cardiovascular calcification is related to intake of starch or sugar in animals [8] and similarly, a high glucose medium enhances calcification of human vascular smooth muscle cells and increases expression of markers 
of bone formation [9]. Also from a recent report citing Cochrane database of trials, excessive fat intake may have an adverse effect on coronary vascular disease (CVD) [10].

Apart from dietary intake, other lifestyle habits may be associated with CAC. A number of studies have reported an association with excessive sleep duration $[11,12]$ and physical activity with carotid or coronary atherosclerosis [13-15]. Also, depression may be a possible risk factor $[16,17]$. It should be noted that these studies also show some inconsistent results.

Previously, we found that composition of dietary macronutrient intake was not associated with a prevalence of coronary artery calcification in healthy Korean adults [18]. The conclusion, however, may not have been definitive as the study was from a cross-sectional analysis. In this study, we hypothesized that the daily intake of macronutrients such as carbohydrate, protein and fat and physical activity or depressive symptoms may show a correlation with progression of CAC in healthy population at low risk of CVD.

\section{Methods}

\section{Study population}

The participants consisted of individuals older than 18 years who underwent a baseline comprehensive health examination at Kangbuk Samsung Hospital Health Screening Center in Seoul and Suwon, South Korea, between 2010 and 2012 and had follow up at 2013. A total of 2623 individuals were identified to have received non-enhanced coronary computed tomography, completed the food frequency questionnaire (FFQ) in addition to questionnaires for depressive symptoms and physical activity at baseline and follow up. The following participants were excluded from analysis: 379 subjects with missing data (on smoking, exercise, and alcohol intake), 44 subjects with a history of cancer, and 52 subjects with a history of cardiovascular disease. As some individuals met more than one criterion for exclusion, the total number of eligible subjects for the study was 2175. Among these subjects we could obtain the answer for questionnaires; 1099 subjects for total energy intake, 917 subjects for composition of macronutrients, 907 subjects for sleep duration and 864 subjects for depression. In Korea, the Industrial Safety and Health Law requires employees to participate in annual or biannual health examinations. Approximately $60 \%$ of the participants were employees of various companies and local governmental organizations.

This study was approved by the Institutional Review Board (IRB) of Kangbuk Samsung Hospital and informed consent requirement was waived as all personal identifiable information was removed prior to accession.

\section{Measurements}

\section{Anthropometric measurements and general characteristics}

Body weight and height of subjects were measured to the nearest $0.1 \mathrm{~kg}$ and $0.1 \mathrm{~cm}$, respectively. Body mass index (BMI) was calculated as weight in kilograms divided by the square of the height in meters. Obesity was defined as BMI $\geq 25 \mathrm{~kg} / \mathrm{m}^{2}$. Data on past medical history, medication use, and health-related behaviors were obtained by a self-administered questionnaire. Questionnaires were used to evaluate education level, smoking status (current or non-current), alcohol consumption (frequency per week, amount) and sleep duration (hours per day). Depression was evaluated by CES-DK score [19] and food frequency data was calculated by CANpro 4.0 (Korean Nutrition Society 2010).

Alcohol intake was examined as unit per day of alcohol consumption. Smokers were divided into two groups: current smoker and non-current smoker. Physical activity was evaluated using the Korean version of the International Physical Activity Questionnaire (IPAQ) short form [20, 21]. The number of days per week and time spent walking per day, as well as moderate and vigorous activities were recorded. The collected data were converted to metabolic equivalent scores (METS) for each type of activity. By multiplying the time engaged in the activity in a week with consideration to the number of METs, metabolic equivalent task minutes per week, MET-min/week, were calculated according to the IPAQ scoring protocol [22]. Blood pressure was measured with electronic sphygmomanometer in the seated position with more than $5 \mathrm{~min}$ of resting prior to the measuring.

\section{Coronary Artery Calcification (CAC) measurement}

Coronary artery calcification (CAC) was detected by a LightSpeed VCT XTe-64 slice MDCT scanner (GE Healthcare, Tokyo, Japan) with the same standard scanning protocol using 2.5-mm thickness, $400 \mathrm{~ms}$ rotation time, $120 \mathrm{kV}$ tube voltage, and $124 \mathrm{mAs}(310 \mathrm{~mA}$ * $0.4 \mathrm{~s})$ tube current under ECG-gated dose modulation. Coronary artery calcification was defined as more than three contiguous pixels above a CT density of 130 Hounsfield Units. The total CAC score was calculated by Agatston's method [23]. Subjects were classified into two subgroups according to CAC score: CAC group $(\mathrm{CAC}$ score $>0)$ or non-CAC group (CAC score $\leq 0)$ by referring to previous studies.

\section{Biochemical marker}

Blood samples were taken from the antecubital vein, collected in serum-separating tube (SST) after at least $10 \mathrm{~h}$ of fasting. Serum levels of total cholesterol and triglyceride were determined using an enzymatic colorimetric assay; low-density lipoprotein cholesterol (LDL-C) and high-density lipoprotein cholesterol (HDL-C) levels were 
directly measured using a homogeneous enzymatic colorimetric assay. Serum fasting glucose level was measured using the hexokinase method. Fasting serum glucose, total cholesterol, LDL-C, HDL-C, triglyceride (TG), alanine aminotransferase (ALT), gamma-glutamyl transferase (GGT), were measured using Bayer Reagent Packs in an automated chemistry analyzer (Advia 1650 Auto analyzer; Bayer Diagnostics, Leverkusen, Germany). High sensitivity C-reactive protein (hsCRP) was analyzed by particle-enhanced immunonephelometry with the BNII System (Dade Behring, Marburg, Germany). All hematologic measurements were analyzed in one laboratory with the same machines by trained staff using the same methodology throughout. The Korean Society of Laboratory Medicine (KSLM) biannually certified the Laboratory Medicine Department at Kangbuk Samsung Hospital in Seoul, Korea for the Korean Association of Quality Assurance for Clinical Laboratories (KAQACL) and the CAP (Collage of American Pathologists) Proficiency Testing designations.

\section{Nutrient intake measurements}

Self-administered food frequency questionnaire (FFQ) was used to obtain nutrient intake data which was designed and validated for use in Korea. Food frequency was estimated by 9 scales (never, 1 time/month, $2-3$ times/month, $1-2$ times/week, $3-4$ times/week, $5-6$ times/week, 1 time/day, 2 times/day and 3 times/day) and portion size was estimated by three scales (half dish, one dish, one and a half dish) for consumption of 103 food items over the past year. Nutrient intake data include total energy $(\mathrm{kcal})$, carbohydrate $(\mathrm{g})$, protein $(\mathrm{g})$ and fat (g). The Food Composition Table, a nutrient database produced by the Korean Nutrition Society to convert food intake into nutrients, was used to perform nutrient analysis [24]. The contribution of each macronutrient to energy was calculated as the ratio of energy from each macronutrient to total energy: percentage of carbohydrate from total energy intake (\%), percentage of fat from total energy intake (\%) and percentage of protein from total energy intake (\%). Food frequency data was calculated by CAN-pro 4.0 (Korean Nutrition Society 2010). Subjects were categorized into four groups according to each macronutrient intake $(\mathrm{Q} 1 \sim \mathrm{Q} 4)$.

\section{Statistical analyses}

Normally distributed variables are presented as the mean $\pm \mathrm{SD}$ and skewed variables are presented as the median (interquartile range). Continuous variables were compared using independent $t$-test between CAC change $\leq 0$ group and CAC change $>0$ group (CAC progression). Categorical variables were expressed as number and percentages then compared between groups using the $x 2$-test. Multiple logistic regression analysis were used to determine Hazard Ratios (HRs) for CAC progression with $95 \%$ confidence intervals (CIs) for quartile groups of each total energy intake, macronutrient intake, physical activity and depression using the lowest quartile group as the reference. To evaluate the significance, two models were constructed. Model 1 was adjusted for age and sex and Model 2 was adjusted for Model 1 and smoking, physical activity, alcohol intake, glucose, triglyceride, HDL-cholesterol, LDL-cholesterol, and blood pressure. P values $<0.05$ were considered statistically significant. The STATA 11.2 software package was used for statistical analysis.

\section{Results}

A total of 2175 participants were divided into two groups according to CAC change and 592 subjects showed CAC progression with CAC change $>0$. Mean follow-up duration was $2.16 \pm 0.15$ years. Baseline characteristics of these groups are illustrated in Table 1. Majority of the participants were male (97\%) and mean age of CAC change $\leq 0$ group and CAC change $>0$ group were $41.6 \pm 5.6$ years and $44.8 \pm 5.5$ years, respectively. CAC progression group were older, with higher BMI, level of obesity, diabetes, hypertension, alcohol intake and adverse lipid profiles (all $p<0.001$ ). However current smoking status did not differ between the groups. Daily total energy intake for CAC change $\leq 0$ group and CAC change $>0$ group were 1512.8 and $1473.7 \mathrm{kcal}$ respectively $(p<0.005)$. However the proportion of carbohydrate, protein and fat intake were not different between the two groups. Mean physical activity of the CAC change $\leq 0$ group and CAC change $>0$ group were 887.5 and 1045.5 METS per day, respectively ( $p=$ 0.02 ) and depression score (CES-D score) were similar with 4 and 5 for each group $(p=0.307)$. We compared the two groups according to quartile of total daily energy intake, each macronutrient intake and physical activity and depressive symptoms (Fig. 1). Total energy, carbohydrate and fat intake showed significant difference between the two groups with $\mathrm{p}$ value of $0.01,0.029$ and 0.016 respectively. However, proportion of protein intake did not vary with $\mathrm{p}$ value of 0.286 . Depressive symptoms and extent of physical activity were similar between the groups. Additional analysis of sleep duration and alcohol intake showed significant difference for alcohol intake $(p=0.001)$ but not for sleep.

As a few macronutrient and alcohol intake showed significant differences, multivariate analysis was conducted in CAC change $>0$ group according to quartiles of total energy, macronutrient intake, physical activity and depressive symptom to confirm relationship between those variables mentioned above and CAC progression. Model 1 and model 2 were suggested with adjustment of possible confounding factors (age, sex, smoking, physical 
Table 1 Baseline characteristics of study participants by CAC change

\begin{tabular}{|c|c|c|c|}
\hline \multirow{3}{*}{ Number } & \multicolumn{2}{|l|}{ CAC change } & \multirow{2}{*}{$\begin{array}{l}\mathrm{p}- \\
\text { value }\end{array}$} \\
\hline & CAC change $\leq 0$ & CAC change $>0$ & \\
\hline & 1,583 & 592 & \\
\hline & $41.6(5.6)$ & $44.8(5.5)$ & $<0.001$ \\
\hline Male (\%) & 93.6 & 99.2 & $<0.001$ \\
\hline $\mathrm{BMI}\left(\mathrm{kg} / \mathrm{m}^{2}\right)$ & 24.9(3.0) & $25.8(3.0)$ & $<0.001$ \\
\hline Obesity (\%) & 45.4 & 56.9 & $<0.001$ \\
\hline Current smoker (\%) & 33.2 & 35.6 & 0.288 \\
\hline Alcohol intake $(\%)^{\mathrm{b}}$ & 32.3 & 40.7 & $<0.001$ \\
\hline High education level $(\%)^{c}$ & 85.5 & 87.0 & 0.404 \\
\hline Diabetes (\%) & 6.3 & 15.0 & $<0.001$ \\
\hline Hypertension (\%) & 20.3 & 33.6 & $<0.001$ \\
\hline Systolic BP $(\mathrm{mmHg})^{a}$ & $118.5(12.2)$ & 121.3(11.9) & $<0.001$ \\
\hline Diastolic BP $(\mathrm{mmHg})^{\mathrm{a}}$ & 75.9(9.4) & $78.1(9.5)$ & $<0.001$ \\
\hline Glucose $(\mathrm{mg} / \mathrm{dL})^{\mathrm{a}}$ & $98.6(15.4)$ & $103.9(21.9)$ & $<0.001$ \\
\hline Total cholesterol $(\mathrm{mg} / \mathrm{dL})^{\mathrm{a}}$ & 206.8(36.4) & 218.6(38.0) & $<0.001$ \\
\hline $\mathrm{LDL}-\mathrm{C}(\mathrm{mg} / \mathrm{dL})^{\mathrm{a}}$ & $130.0(32.7)$ & $141.1(34.2)$ & $<0.001$ \\
\hline $\mathrm{HDL}-\mathrm{C}(\mathrm{mg} / \mathrm{dL})^{\mathrm{a}}$ & $51.7(12.4)$ & $49.3(11.1)$ & $<0.001$ \\
\hline Triglycerides $(\mathrm{mg} / \mathrm{dL})^{d}$ & 130(89-190) & 153(110.5-215) & $<0.001$ \\
\hline $\operatorname{ALT}(U /)^{d}$ & $25(18-37)$ & $28(20-40)$ & $<0.001$ \\
\hline GTP $(U / I)^{d}$ & $34(22-54)$ & $40(27-65)$ & $<0.001$ \\
\hline C-reactive protein $(\mathrm{mg} / \mathrm{L})^{\mathrm{d}}$ & $0.06(0.03-0.11)$ & $0.07(0.04-0.13)$ & 0.022 \\
\hline Total energy & 1530.9(1055.0-1933.0) & 1483.5(659.4.2-1788.9) & 0.001 \\
\hline Carbohydrate \% & $74.7(69.0-80.0)$ & $76.0(68.6-80.3)$ & 0.497 \\
\hline Protein\% & $13.8(12.5-15.2)$ & $13.9(12.6-15.0)$ & 0.966 \\
\hline Fat $\%$ & 16.3(12.7-20.3) & $14.9(12.3-20.2)$ & 0.244 \\
\hline Physical activity (MET-min/week) & $887.5(396-1836)$ & $1045.5(438-1977)$ & 0.800 \\
\hline Depression (CES-D score) & $4(1-9)$ & $5(1-9)$ & 0.283 \\
\hline
\end{tabular}

Data are ${ }^{a}$ means (standard deviation), ${ }^{d}$ medians (interquartile range), or percentages

Abbreviations: ALT, alanine aminotransferase; BMI, body mass index; BP, blood pressure; LDL-C, low-density lipoprotein-cholesterol; HDL-C, high-density lipoprotein-cholesterol; GTP, gamma glutamyl transferase

${ }^{b} \geq 20 \mathrm{~g} /$ day; $^{c} \geq$ College graduate

activity, alcohol intake, glucose, triglyceride, HDLcholesterol, LDL-cholesterol, and blood pressure) (Tables 2, 3). However, after adjustment of possible confounding factors, the HRs for CAC change $>0$ were not significant in all categories with insignificant $\mathrm{p}$ values for trends in both models. We conducted a sub-analysis only including those with more than two years of follow up however it did not alter the result (Additional file 1).

\section{Discussion}

In this relatively healthy cohort of Korean adults, total energy intake, proportion of carbohydrate, protein, fat, physical activity and depression were not associated with progression of CAC after adjustment of possible confounding factors.
A few studies have investigated the relationship between macronutrient intake and $\mathrm{CAC}$ or other markers of subclinical atherosclerosis $[8,18,25]$. Recent report from Cochrane database showed excessive fat intake has an adverse effect on coronary vascular disease (CVD) [10]. On the other hand, a large cross-sectional population-based study reported that healthy dietary pattern was not related to CAC with carotid artery IMT after adjustment for various confounders [25]. Recently, we also suggested that the prevalence of CAC may not be associated with composition of macronutrient intake [18]. Although previous studies have studied the relationship between macronutrient intake and $\mathrm{CAC}$, to the best of our knowledge, our study is the first to analyze the relationship between macronutrient intake and progression of CAC. An important finding in our study is 

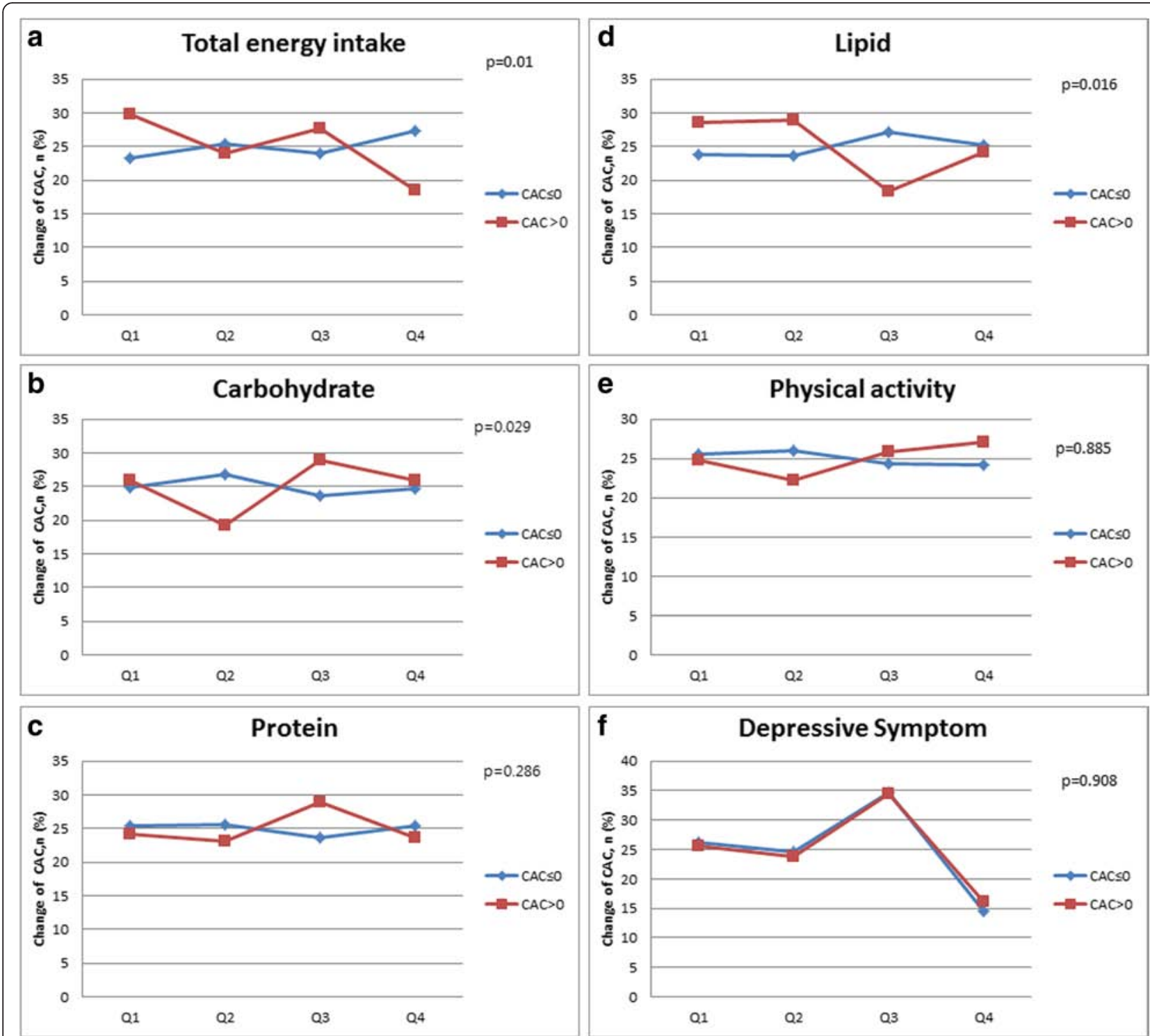

Fig. 1 Associations of total energy, macronutrients, physical activity and depression according to coronary artery calcium change. a Correlation between CAC change and total E intake. $\mathbf{b}$ Correlation between CAC change and carbohydrate intake. $\mathbf{c}$ Correlation between CAC change and protein intake. $\mathbf{d}$ Correlation between CAC change and lipid intake. e Correlation between CAC change and physical activity. $\mathbf{f}$ Correlation between CAC change and depression

that high carbohydrate and fat diet did not affect CAC progression. This result is the same as our previous study [18].

There are several published data on a potential benefit of physical activity on atherosclerosis [13, 14, 26]. A population-based study found that vigorous physical activity had an inverse association with incident CAC [13] and a prospective study reported that maintaining regular high physical activity level might protect against atherosclerosis [14].

From a study of a small number of spinal cord injury (SCI) cases, able-bodied SCI patients had improved common carotid artery intima-media thickness (IMT) compared with sedentary SCI [26]. Several studies have also revealed a positive effect of physical activity on reducing level of many atherosclerotic factors such as CRP and cytokines [27]. However, our study failed to show a significant association between physical activity and progression of atherosclerosis. Although the overall association between CAC progression and physical activity was found to be insignificant, there were a positive trend of higher level of physical activity in CAC change $>0$ group whereas physical activity tended to be lower in $C A C$ change $\leq 0$ group. One possible explanation is that as the BMI of CAC change $>0$ group was higher than that of the other 
Table 2 Multivariate analysis for coronary artery calcification score according to quartile of macronutrients intake

\begin{tabular}{|c|c|c|c|c|c|}
\hline & Q1 & Q2 & Q3 & Q4 & $P$ for trend \\
\hline \multicolumn{6}{|l|}{ Total energy } \\
\hline$N($ total /CAC change $>0$ ) & $275 / 87$ & $275 / 70$ & $275 / 81$ & $274 / 54$ & \\
\hline Model $1^{\mathrm{a}}$ & 1.00 & $0.73(0.50-1.08)$ & $1.01(0.69-1.48)$ & $0.71(0.47-1.08)$ & 0.317 \\
\hline Model $2^{b}$ & 1.00 & $1.09(0.61-1.94)$ & $1.25(0.70-2.22)$ & $1.02(0.56-1.87)$ & 0.826 \\
\hline \multicolumn{6}{|l|}{ Carbohydrate \% } \\
\hline N (total /CAC change $>0$ ) & $230 / 59$ & $229 / 44$ & $229 / 66$ & $229 / 59$ & \\
\hline Model $1^{a}$ & 1.00 & $0.67(0.42-1.06)$ & $1.12(0.72-1.73)$ & $0.79(0.50-1.23)$ & 0.764 \\
\hline Model $2^{b}$ & 1.00 & $0.63(0.34-1.18)$ & $1.58(0.86-2.91)$ & $0.90(0.48-1.68)$ & 0.581 \\
\hline \multicolumn{6}{|l|}{ Protein\% } \\
\hline N (total /CAC change $>0$ ) & $230 / 55$ & $229 / 53$ & $229 / 66$ & $229 / 54$ & \\
\hline Model $1^{a}$ & 1.00 & $0.99(0.63-1.56)$ & $1.42(0.92-2.21)$ & $0.89(0.57-1.41)$ & 0.951 \\
\hline Model $2^{b}$ & 1.00 & $1.09(0.58-2.07)$ & $1.18(0.63-2.21)$ & $0.68(0.36-1.27)$ & 0.252 \\
\hline \multicolumn{6}{|l|}{ Fat $\%$} \\
\hline N (total /CAC change $>0$ ) & $230 / 55$ & $229 / 53$ & 229/66 & $229 / 54$ & \\
\hline Model $1^{\mathrm{a}}$ & 1.00 & $1.32(0.85-2.03)$ & $0.75(0.47-1.20)$ & $1.07(0.69-1.68)$ & 0.658 \\
\hline Model $2^{\mathrm{b}}$ & 1.00 & $1.88(1.01-3.51)$ & $0.58(0.30-1.13)$ & $0.99(0.52-1.86)$ & 0.238 \\
\hline
\end{tabular}

Data are adjusted hazard ratios with $95 \% \mathrm{Cl}$

${ }^{a}$ Model 1: Adjusted for age and sex. ${ }^{\mathrm{b}}$ Model 2: Model 1+ smoking, physical activity, alcohol intake, glucose, triglyceride, HDL-cholesterol, LDL-cholesterol, waist circumference, blood pressure

group, the individuals may have tried to be more active and lose more weight.

Cardiovascular disease (CVD) and comorbid depression seem to have a bidirectional relationship. Previous etiological and prognostic studies have suggested that depression may worsen the severity of CVD and also contribute to the onset of the disease, thus impacting CVD in multiple ways $[16,17]$. The interaction of CVD and depression may involve experience of chronic stress triggering neurohormonal activation, autonomic neurocardiac dysfunction with the ensuing cytokine cascades leading to chronic inflammation [28]. Despite the previous studies, our result with depressive symptoms failed to show a significant association, which may be explained as follows. CES-D scale, which is designed to measure depressive symptoms in general population, has more than 16 points considered to be depressive in Western population [29]. However the cutoff for Koreans is considered to be 21 points and higher than Western population [19] and may be due to the sociocultural differences. Moreover, while women tend to respond to stress with higher anxiety and depressiveness, men are more reluctant to report emotional distress. In our study population only $15 \%$ showed more than 10 points, which meant that the majority were nondepressive subjects, and $95 \%$ were male. This uneven sampling may have underpowered our analysis.

This study has several limitations. First of all, this study evaluated CAC change over two years of follow up but as atherosclerotic calcification of vessels may require

Table 3 Multivariate analysis for coronary artery calcification score according to physical activity and depression

\begin{tabular}{|c|c|c|c|c|c|}
\hline & Q1 & Q2 & Q3 & Q4 & $P$ for trend \\
\hline \multicolumn{6}{|l|}{ Physical activity } \\
\hline N (total /CAC change >0) & $270 / 70$ & $266 / 63$ & $263 / 73$ & $265 / 76$ & \\
\hline Model $1^{a}$ & 1.00 & $0.90(0.60-1.36)$ & $1.00(0.67-1.50)$ & $1.01(0.68-1.52)$ & 0.833 \\
\hline Model $2^{b}$ & 1.00 & $0.99(0.56-1.74)$ & $1.12(0.63-1.98)$ & $1.04(0.58-1.85)$ & 0.894 \\
\hline \multicolumn{6}{|l|}{ Depression } \\
\hline N (total /CAC change > 0) & $225 / 64$ & $211 / 59$ & 299/86 & $129 / 40$ & \\
\hline Model $1^{\mathrm{a}}$ & 1.00 & $0.92(0.60-1.43)$ & $0.90(0.60-1.35)$ & $1.09(0.66-1.79)$ & 0.927 \\
\hline Model $2^{\mathrm{b}}$ & 1.00 & $1.57(0.83-2.99)$ & $0.93(0.51-1.71)$ & $1.66(0.83-3.33)$ & 0.478 \\
\hline
\end{tabular}

Data are adjusted hazard ratios with $95 \% \mathrm{Cl}$

${ }^{a}$ Model 1: Adjusted for age and sex. ${ }^{\mathrm{b}}$ Model 2: Model 1+ smoking, physical activity, alcohol intake, glucose, triglyceride, HDL-cholesterol, LDL-cholesterol, blood pressure 
a long-standing pathological process, two year of observation might be too short to find any change in CAC. This cohort consisted of relatively healthy individuals, these results cannot be easily applied to other populations. In addition, in the study there may be a selection bias from having healthy workers. We defined CAC progression as a CAC change of $>0$, and this includes onepoint changes. However, a one-point increase in CAC score is not clinically meaningful and the extent of CAC increases differ according to the baseline CAC score [30]. A subtype analysis may help to elucidate a more clear association. Also as data for food intake was via a self-administered questionnaire, it is not free of recall bias and measurement error. However, this type of bias would be non-differential, biasing the estimate towards the null. Finally, we did not have information on the intake of macronutrients subtypes, as the types of fatty acids (saturated vs. unsaturated) and types of carbohydrate (complex vs. simple sugars) may differently affect preclinical atherosclerosis.

\section{Conclusions}

In summary, the presented data showed that levels of macronutrient intake, physical activity, depressive symptoms were not associated with progression of CAC. Despite several limitations, this study is strengthened by having a large cohort of relatively healthy Korean adults. To our knowledge, this is the first study that investigates associations between macronutrient intake and progression of CAC in Koreans. Further research is needed to confirm our findings.

\section{Additional file}

Additional file 1: Multivariate hazard ratios (HRs) for CAC score according to quartile of macronutrients intake (two year follow up data). (DOCX $19 \mathrm{~kb}$ )

\section{Abbreviations}

ALT: alanine aminotransferase; BMI: body mass index; CAC: coronary artery calcium; CES-D: center for epidemiology studies-depression; CVD: coronary vascular disease; DBP: diastolic blood pressure; FFQ: food frequency questionnaire; GTP: gamma glutamyl transferase; HDL-C: high-density lipoprotein cholesterol; hsCRP: high sensitivity C-reactive protein.; IPAQ: International Physical Activity Questionnaire; LDL-C: low-density lipoprotein cholesterol; MDCT: multidetector computed tomography; METS: metabolic equivalent scores; SBP: systolic blood pressure; TG: triglyceride.

\section{Competing interests}

All the authors declare that: 1) the paper is not under consideration elsewhere; 2) none of the paper's contents have been previously published; 3) all authors have read and approved the manuscript; 4) there is full disclosure of any relationship with industry; and 5) all authors have no relevant conflicts of interest.

\section{Authors' contributions}

K.S takes full responsibility for the data collection and integrity of the analyses. E.C contributed to the hypothesis, wrote the manuscript. J.L, S.L, J.K,
B.K $K^{1}$ and $B . K^{2}$ reviewed/edited the manuscript and contributed to the discussion section. K.S is the guarantor for the article and all authors have read and agree with the manuscript as written.

\section{Acknowledgments}

We acknowledge the efforts of the health-screening group at Kangbuk Samsung Hospital, Korea. This study was partially funded by the Korean society of hypertension in 2014

Received: 11 August 2015 Accepted: 8 January 2016

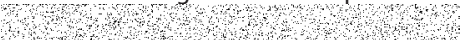

\section{References}

1. Rifkin RD, Parisi AF, Folland E. Coronary calcification in the diagnosis of coronary artery disease. Am J Cardiol. 1979;44:141-7.

2. Stary HC, Chandler AB, Dinsmore RE, Fuster V, Glagov S, Insull Jr W, et al. A definition of advanced types of atherosclerotic lesions and a histological classification of atherosclerosis. A report from the Committee on Vascular Lesions of the Council on Arteriosclerosis, American Heart Association. Arterioscler Thromb Vasc Biol. 1995;15:1512-31.

3. McEvoy JW, Blaha MJ, Defilippis AP, Budoff MJ, Nasir K, Blumenthal RS, et al. Coronary artery calcium progression: an important clinical measurement? A review of published reports. J Am Coll Cardiol. 2010;56:1613-22.

4. Cassidy-Bushrow AE, Bielak LF, Sheedy 2nd PF, Turner ST, Kullo IJ, Lin X, et al. Coronary artery calcification progression is heritable. Circulation. 2007; 116:25-31.

5. Kramer CK, von Muhlen D, Gross JL, Laughlin GA, Barrett-Connor E. Blood pressure and fasting plasma glucose rather than metabolic syndrome predict coronary artery calcium progression: the Rancho Bernardo Study. Diabetes Care. 2009:32:141-6.

6. Elkeles RS, Godsland IF, Rubens MB, Feher MD, Nugara F, Flather MD. The progress of coronary heart disease in Type 2 diabetes as measured by coronary calcium score from electron beam computed tomography (EBCT): the PREDICT study. Atherosclerosis. 2008;197:777-83.

7. Kronmal RA, McClelland RL, Detrano R, Shea S, Lima JA, Cushman M, et al. Risk factors for the progression of coronary artery calcification in asymptomatic subjects: results from the Multi-Ethnic Study of Atherosclerosis (MESA). Circulation. 2007;115:2722-30.

8. Zhou YB, Zhang J, Cai Y, Teng X, Duan XH, Song JQ, et al. Insulin resistance induces medial artery calcification in fructose-fed rats. Exp Biol Med (Maywood). 2012;237:50-7.

9. Liu F, Zhong H, Liang JY, Fu P, Luo ZJ, Zhou L, et al. Effect of high glucose levels on the calcification of vascular smooth muscle cells by inducing osteoblastic differentiation and intracellular calcium deposition via BMP-2/ Cbfalpha-1 pathway. J Zhejiang Univ Sci B. 2010;11:905-11.

10. Rees K, Dyakova M, Wilson N, Ward K, Thorogood M, Brunner E. Dietary advice for reducing cardiovascular risk. Cochrane Database Syst Rev. 2013; 12, CD002128.

11. Abe T, Aoki T, Yata S, Okada M. Sleep duration is significantly associated with carotid artery atherosclerosis incidence in a Japanese population. Atherosclerosis. 2011;217:509-13.

12. Ma CC, Burchfiel CM, Charles LE, Dorn JM, Andrew ME, Gu JK, et al. Associations of objectively measured and self-reported sleep duration with carotid artery intima media thickness among police officers. Am J Ind Med. 2013;56:1341-51.

13. Delaney JA, Jensky NE, Criqui MH, Whitt-Glover MC, Lima JA, Allison MA The association between physical activity and both incident coronary artery calcification and ankle brachial index progression: the multi-ethnic study of atherosclerosis. Atherosclerosis. 2013;230:278-83.

14. Kwasniewska M, Jegier A, Kostka T, Dziankowska-Zaborszczyk E, Rebowska E, Kozinska J, et al. Long-term effect of different physical activity levels on subclinical atherosclerosis in middle-aged men: a 25-year prospective study. PLoS One. 2014;9, e85209.

15. Ried-Larsen M, Grontved A, Kristensen PL, Froberg K, Andersen LB. Moderate-and-vigorous physical activity from adolescence to adulthood and subclinical atherosclerosis in adulthood: prospective observations from the European Youth Heart Study. Br J Sports Med. 2015:49:107-12.

16. Hamer M, Kivimaki M, Lahiri A, Marmot MG, Steptoe A. Persistent cognitive depressive symptoms are associated with coronary artery calcification. Atherosclerosis. 2010;210:209-13. 
17. Rozanski A, Blumenthal JA, Davidson KW, Saab PG, Kubzansky L The epidemiology, pathophysiology, and management of psychosocial risk factors in cardiac practice: the emerging field of behavioral cardiology. J Am Coll Cardiol. 2005:45:637-51.

18. Sung KC, Sung E, Byrne CD, Kim YB, Ahn CW, Chung HK. Composition of dietary macronutrient intake is not associated with prevalence of coronary artery calcification in healthy Korean adults. Ann Nutr Metab. 2015;66:36-43.

19. Cho MJ, Kim KH. Use of the Center for Epidemiologic Studies Depression (CES-D) Scale in Korea. J Nerv Ment Dis. 1998;186:304-10.

20. Oh JYYY, Kim BS, Kang JH. Validity and reliability of Korean version of International Physical Activity Questionnaire (IPAQ) short form. J Korean Acad Fam Med. 2007;28:532-41.

21. Chun MY. Validity and reliability of korean version of international physical activity questionnaire short form in the elderly. Korean J Fam Med. 2012;33:144-51.

22. Craig CL, Marshall AL, Sjostrom M, Bauman AE, Booth ML, Ainsworth BE, et al. International physical activity questionnaire: 12-country reliability and validity. Med Sci Sports Exerc. 2003;35:1381-95.

23. Agatston AS, Janowitz WR, Hildner FJ, Zusmer NR, Viamonte Jr M, Detrano R. Quantification of coronary artery calcium using ultrafast computed tomography. J Am Coll Cardiol. 1990;15:827-32.

24. Ahn Y, Kwon E, Shim JE, Park MK, Joo Y, Kimm K, et al. Validation and reproducibility of food frequency questionnaire for Korean genome epidemiologic study. Eur J Clin Nutr. 2007;61:1435-41.

25. Nettleton JA, Schulze MB, Jiang R, Jenny NS, Burke GL, Jacobs Jr DR A priori-defined dietary patterns and markers of cardiovascular disease risk in the Multi-Ethnic Study of Atherosclerosis (MESA). Am J Clin Nutr. 2008;88:185-94.

26. Matos-Souza JR, Silva AA, Campos LF, Goulart D, Schreiber R, de Rossi G, et al. Physical activity is associated with improved subclinical atherosclerosis in spinal cord injury subjects independent of variation in traditional risk factors. Int J Cardiol. 2013;167:592-3.

27. Astengo M, Dahl A, Karlsson T, Mattsson-Hulten L, Wiklund O, Wennerblom B. Physical training after percutaneous coronary intervention in patients with stable angina: effects on working capacity, metabolism, and markers of inflammation. Eur J Cardiovasc Prev Rehabil. 2010;17:349-54.

28. Moller-Leimkuhler AM. Higher comorbidity of depression and cardiovascular disease in women: a biopsychosocial perspective. World J Biol Psychiatry. 2010;11:922-33.

29. LS R. The CES-D Scale: A self report depression scale for research in the general population. Appl Psychol Meas. 1977:1:385-401.

30. Kestenbaum BR, Adeney KL, de Boer IH, IX JH, Shlipak MG, Siscovick DS. Incidence and progression of coronary calcification in chronic kidney disease: the Multi-Ethnic Study of Atherosclerosis. Kidney Int. 2009;76:991-8.

\section{Submit your next manuscript to BioMed Central and we will help you at every step:}

- We accept pre-submission inquiries

- Our selector tool helps you to find the most relevant journal

- We provide round the clock customer support

- Convenient online submission

- Thorough peer review

- Inclusion in PubMed and all major indexing services

- Maximum visibility for your research

Submit your manuscript at www.biomedcentral.com/submit

C Biomed Central 